\title{
EDITORIAL
}

\section{The Continuing Confusion in Figuring out the Surname of a Chinese Author - A Proposed Solution}

\author{
Tsung O. CHENG
}

Confusion continues in figuring out which of the Chinese author's name in a publication is the surname, although I have made numerous attempts in suggesting possible solutions to this nasty problem in the past. ${ }^{(1-10)}$ There is a fundamental difference between a Chinese name and a Western name. A Western name places the given name first and surname last, for example, former American president George Bush. On the other hand, a Chinese name places the surname first followed by the given name, for example, Chinese president Hu Jintao, just as in the telephone directory. The reason behind this tradition is that, in the Chinese culture, people hold great reverence for their origins and ancestors. So, part of this process means that people honor their ancestors by placing the family names ahead of the given names. Thus the name of the founding father of the People's Republic of China is Mao Tse Tung (now spelled Mao Zedong). I have often seen articles in American newspapers, magazines or journals in which Mao would be erroneously addressed as Mr. Tung or Mr. Zedong, thus creating a great deal of confusion.

The difference in name order between the Chinese custom and Western culture may cause a serious problem when a name list in any social function is created in an alphabetical order, as is customarily done. For example, during the state visit of Chinese president $\mathrm{Hu}$ Jintao in January 2011, the guest list for the state dinner released by the White House had the name of the Chinese foreign minister Yang Jiechi listed following Valerie Jarrett in the middle of the list (Figure 1), instead of toward the end of the list where $Y$ in Yang's surname should appear just before the last alphabet $Z$.

This state of confusion often exists in the registration directories of various medical conventions. I oftentimes had considerable difficulties in locating the whereabouts of my Chinese colleagues who were known to be attending these meetings, because their names were listed in an erroneous alphabetical order

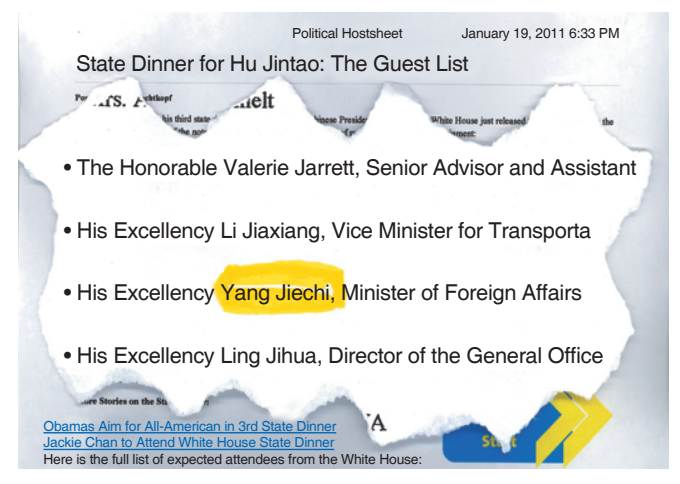

Figure 1. Portion of the guest list released by the White House for the state dinner for Chinese president Hu Jintao in January 2011, in which the name of Chinese foreign minister Yang Jiechi was listed erroneously following the Honorable Valerie Jarrett, instead of toward the end where the alphabet $Y$ in Yang's surname should appear.

by their given name instead of their surnames. I soon learned that, in order to locate my colleagues, I must search alphabetically not only by their surnames but also by their given names. I strongly advise my American and European colleagues to do likewise.

Another example of confusion may occur when the Chinese person's name consists of two characters in total. For instance, the recently retired basketball player Yao Ming is sometimes referred to in the Western newsmedia as Mr. Ming, instead of Mr. Yao, which is his surname. Of course, if one is lucky to have the same surname and given name in English, as in the case of the renowned Chinese pianist Lang Lang - even though his surname and given name in Chinese (郎朗) are quite different - there should be no problem in finding him anywhere in the world.

This state of confusion is further compounded by

CThe Chinese Journal of Integrated Traditional and Western Medicine Press and Springer-Verlag Berlin Heidelberg 2012 The Department of Medicine, The George Washington University Medical Center, 2150 Pennsylvania Avenue, N.W., Washington, D.C. 20037, USA; Tel: 202-741-2426, Fax: 202-741-2324, E-mail: tcheng@mfa.gwu.edu

DOI: $10.1007 / \mathrm{s} 11655-012-1061-7$ 
the fact that some Chinese people who emigrate to, or do business with, Western countries oftentimes adopt a Westernized name by simply reversing the 'surname given-name' order to 'given-name surname' ('Ming Yao', to follow the previous example). Or, in my case, although my Chinese name is 鄭宗鋀 (or Cheng Tsung O), I had to reverse it to Tsung $O$. Cheng in order to conform to the Western custom. However, this results in further confusion, because people have oftentimes addressed me as Mr. Tsung or just Tsung. When generation names - these generational names are worked out long in advance, historically in a generation poem - are used as part of a twocharacter given name, it is not only inappropriate but also confusing to use just the first character, because they could just as easily refer to my brothers whose given names are Tsung Pei and Tsung Zhen or my sister whose given name is Tsung Wu. Such mistakes commonly occur in Western societies where the first part of the given name is frequently mistakenly used as the first name when the given name is not hyphenated or adjoined.

The use of a comma between a surname and given name has been suggested in some circles. However, it is acceptable only if the name is in isolation (such as part of an alphabetized list or on a field of a government document) but not as part of a sentence. For example, the sentence "Professor Wang, Xin-fang is the father of modern echocardiography" would be wrong.

Because one of every four persons on the planet is a Chinese, the matter of knowing the surname of a Chinese person becomes extremely important.

Several years ago, someone suggested using initials of Chinese given names to resolve the problem. What a barbarous practice! There is no way to initialize a Chinese character; this is simply a Western alphabetic practice. Who would ever know who ZD Mao or Z.D. Mao or Z-D Mao (for Mao Zedong ) or XP Deng or X.P. Deng or X-P Deng (for Deng Xiaoping) is? This problem exists where Westernized Chinese authors have become used to initials and do not give their full names. We should never initialize a Chinese full name just to follow the Western style. Moreover, to put a hyphen between initials is alien to the Chinese pinyin system.

One possible solution to this problem was initiated by the editors of this journal, in which the authors' surnames were spelled all in capitals and the first and middle names hyphenated. Unfortunately, this method has not been applied to authors from countries outside mainland China. ${ }^{(10)}$ I propose that, for the sake of uniformity and to avoid any possible misunderstanding, all authors' names should have their surnames capitalized to ensure no misrepresentation or misquotation. I am pleased to report that this practice will take effect with this issue of the journal. I hope that other medical and scientific journals all around the world will follow suit too.

\section{REFERENCES}

1. Cheng TO. The Chinese last name. J Thorac Cardiovasc Surg 1988;96:832.

2. Cheng TO. What's in a name - the Chinese name? Angiology 1989;40:324.

3. Cheng TO. Word order: Oriental or occidental? Tex Heart Inst J 1989;16:121.

4. Cheng TO. Use of surnames to identify individuals of Chinese ancestry. Am J Epidemiol 1994;140:76.

5. Cheng TO. Chinese last name comes first. Int $\mathrm{J}$ Cardiol 1995;49:94

6. Cheng TO. Chinese first name comes last. Clin Cardiol 2004;27:A30.

7. Cheng TO. Chinese authors' names should include their names in Chinese. Int J Cardiol 2008;123:169.

8. Cheng TO. Confusion in Chinese authors' names. Int $\mathrm{J}$ Cardiol 2009;134:244.

9. Cheng TO. Chinese personal name variations in medical publications. Implications for information retrieval. Int $\mathrm{J}$ Cardiol 2010;139:211-212.

10. Cheng TO. How to figure out the surname of a Chinese author? Int J Cardiol 2011;140:259-260.

(Received February 1, 2012) Edited by ZHANG Wen 